\title{
Chevron versus scarf osteotomy for 1-2 intermetatarsal reduction in the surgical treatment of hallux valgus: a systematic review and meta-analysis
}

\author{
Simon E Smith ${ }^{1,2^{*}}$, Karl B Landorf ${ }^{1,2}$, Hylton B Menz ${ }^{2}$ \\ From Australasian Podiatry Council Conference 2011 \\ Melbourne, Australia. 26-29 April 2011
}

\section{Background}

Surgical correction for hallux valgus (HV) has been demonstrated to be effective compared with watchful waiting and orthotic therapy, and improves the quality of life of individuals with HV. The severity of the HV deformity generally dictates the type of procedure choice, and it is generally considered that the scarf osteotomy affords a greater reduction of the 1-2 intermetatarsal angle (IMA) than the chevron osteotomy. The objective of this study was to compare the radiographic angular correction of the 1-2 IMA for the chevron versus the scarf osteotomy.

\section{Methods}

A systematic review and meta-analysis was conducted. The following databases were searched to identify English language studies evaluating the chevron and scarf osteotomy: Medline, Embase (Ovid), CINAHL (EBSCO Host), the Cochrane Database of Systematic Reviews and Cochrane Central Register of Controlled Clinical Trials. No date restrictions, previous to November 2010, were applied. Additional hand and electronic content searches of orthopaedic and foot-related journals and text books were performed. Randomised controlled trials, and prospective and retrospective cohort studies were included in the analysis. The mean and standard deviation for pre- and post-operative 1-2 IMA for included studies were entered into Review Manager for analysis. The pooled mean difference (in degrees) and 95\% confidence intervals (95\% CIs) for the 1-2 IMA

\footnotetext{
* Correspondence: simon@smithfootankle.com.au

'Department of Podiatry, Faculty of Health Sciences, La Trobe University, Bundoora, Victoria, Australia

Full list of author information is available at the end of the article
}

were compared between categories (i.e. chevron versus scarf osteotomy).

\section{Results}

There were 24 studies meeting the inclusion criteria for the chevron osteotomy and 8 studies for the scarf osteotomy, involving a total of 1303 patients. The pooled mean difference and $95 \%$ CIs for the chevron and scarf osteotomies were $5.30 \pm 0.21$ degrees (5.09, $5.51)$ and $6.21 \pm 0.51$ degrees $(5.70,6.72)$, respectively.

\section{Conclusions}

The scarf osteotomy produces a marginally greater reduction in the 1-2 IMA compared with the chevron osteotomy. However, the included studies were of moderate to poor methodological quality, somewhat limiting the confidence of this result. In addition, our review did not analyse the post-operative clinical outcomes of these two procedures. There is therefore a need for more well designed prospective cohort and randomized controlled trials to compare the angular correction and clinical outcomes of the chevron and scarf osteotomy.

\section{Author details \\ ${ }^{1}$ Department of Podiatry, Faculty of Health Sciences, La Trobe University, Bundoora, Victoria, Australia. ${ }^{2}$ Musculoskeletal Research Centre, Faculty of} Health Sciences, La Trobe University, Bundoora, Victoria, Australia.

Published: 20 May 2011

doi:10.1186/1757-1146-4-S1-O44

Cite this article as: Smith et al:: Chevron versus scarf osteotomy for 1-2 intermetatarsal reduction in the surgical treatment of hallux valgus: a systematic review and meta-analysis. Journal of Foot and Ankle Research 2011 4(Suppl 1):044.

\section{C)

(c) 2011 Smith et al; licensee BioMed Central Ltd. This is an open access article distributed under the terms of the Creative Commons Attribution License (http://creativecommons.org/licenses/by/2.0), which permits unrestricted use, distribution, and reproduction in any medium, provided the original work is properly cited. 